\title{
Herlyn-Werner-Wunderlich Sendromu
}

Mine ISLIMYE TAȘKIN, ${ }^{a}$

Erdoğan BÜLBÜL,

Eda ÜREYEN, ${ }^{a}$

Ertan ADALI ${ }^{a}$

${ }^{a}$ Kadın Hastalıkları ve Doğum AD,

${ }^{\mathrm{b}}$ Radyoloji AD,

Balıkesir Üniversitesi Tıp Fakültesi, Balıkesir

Geliş Tarihi/Received: 12.03 .2015

Kabul Tarihi/Accepted: 13.05.2015

Yazışma Adresi/Correspondence: Mine ISLIMYE TAȘKIN

Balıkesir Üniversitesi Tıp Fakültesi, Kadın Hastalıkları ve Doğum AD, Balıkesir, TÜRKIYE/TURKEY

minetaskin1302@yahoo.com.tr

Bu çalıșmanın özeti, 12. Uludağ Jinekoloji ve Obstetri Kı̧̧ Kongresi (05-08 Mart 2015, Bursa)'nde poster olarak sunulmuştur.

\section{Herlyn-Werner-Wunderlich Syndrome: Case Report}

ÖZET Herlyn-Werner-Wunderlich sendromu (HWWS), Müllerian kanal anomalilerinin nadir görülen bir varyantıdır. Klasik triadı: kör hemivajina, uterin didelfis ve ipsilateral renal agenezidir. Klinik prezentasyonu genellikle menarş ile başlayan, progresif olarak artan ve hematokolposa sekonder gelişen şiddetli dismenoredir. Sebebi ve etiyopatogenezi bilinmemekte birlikte, erken tanı ve tedavi komplikasyonların önlenmesi ve fertilitenin korunmasında önemlidir. Bu çalışmada, dış merkezde pelvik kitle saptanması nedeni ile kliniğimize refere edilen, dismenore öyküsü olan, 18 yaşındaki bekâr olguda tanısını manyetik rezonans görüntüleme (MRG) ile koyduğumuz HWWS sunulmuştur. Bu sendrom şiddetli dismenore, pelvik kitle ve renal agenezi ile başvuran genç kadınlarda akla gelmelidir. MRG, HWWS'nun tanısında çok değerli bir görüntüleme yöntemidir. Vajinal septumun rezeksiyonu hem ağrının ortadan kaldırılmasında hem de komplikasyonların önlenmesinde en uygun tedavi yöntemidir.

Anahtar Kelimeler: Hematokolpos; manyetik rezonans görüntüleme; kalıtsal renal agenezis; endometriyoz

ABSTRACT Herlyn-Werner-Wunderlich syndrome (HWWS) is a rare variant of Müllerian duct anomalies. The classic triad of this syndrome: obstructed hemivagina, didelphys uterus and ipsilateral renal agenesis. It typically presents with severe and progressive dysmenorrhea starting after menarche that was determined secondary to hematocolpos. Although the exact cause and pathogenesis of the syndrome are uncertain; the diagnosis and treatment at an early stage can prevent complications and preserve fertility. Herein we describe the case of 18-year-old who presented with dysmenorrhea and pelvic mass and that we confirmed the diagnosis with magnetic resonance imaging (MRI). When unilateral renal agenesis, severe dysmenorrhea and pelvic mass coexist in a young girl, physicians should remember HWWS. MRI is a very suitable diagnostic tool in order to perform the correct diagnosis. Vaginal septum resection is the best treatment method for reducing pain and preventing the complications.

Keywords: Hematocolpos; magnetic resonance imaging; hereditary renal agenesis; endometriosis

$\mathrm{H}$ erlyn-Werner-Wunderlich sendromu (HWWS), Müllerian kanal anomalilerinin nadir görülen bir varyantıdır; uterin didelfis, kör hemivajina ve ipsilateral renal agenezi ile seyretmektedir. Obstrükte hemivajina, ipsilateral renal agenezi baş harflerinden oluşan $\mathrm{OH}$ VIRA sendromu olarak da adlandırılmaktadır. İlk kez 1922 yılında rapor edilmiştir. Gerçek insidansı bilinmemekle birlikte tahmini olarak \%0,1-3,8 arasındadır. ${ }^{1}$ HWWS’nin, gebeliğin yaklaşık 9. haftasında Müllerian kanal- 
ların lateral ve vertikal füzyon anomalisine bağlı olarak geliştiği düşünülmektedir. ${ }^{2}$ Klinik prezentasyonu genellikle menarş ile başlayan, progresif olarak artan ve hematokolposa sekonder olarak gelişen şiddetli dismenoredir. Kör hemivajina tanısı konulmadığ zaman endometriyoz, pelvik inflamatuar hastalık, komplike ovaryan kistler gibi diğer pelvik hastalıklardan ayırıcı tanı yapılması zor olabilmektedir. Öncelikli olarak Müllerian kanal anomalilerinin tanısında ultrasonografi (USG) ilk tercih olmasına rağmen kesin tanı için manyetik rezonans görüntüleme (MRG) önerilmektedir. ${ }^{3}$

Bu çalışmada diş merkezde pelvik kitle saptanması nedeni ile kliniğimize refere edilen, dismenore öyküsü olan, 18 yaşındaki bekâr olguda tanısını MRG ile koyduğumuz HWWS sunulmuştur.

\section{OLGU SUNUMU}

On sekiz yaşındaki bekâr olgu; ağrllı adet görme ve dış merkezde yapılan USG'de kist saptanması nedeni ile üniversitemiz kadın hastalıkları ve doğum polikliniğine başvurdu. Olgunun dismenore şikâyeti vardı ve adetleri düzenliydi. Son menstrüasyon tarihi üç hafta önce idi. Nisan 2014 tarihinde adneksiyal kitle nedeni ile sol salpingoooferektomi yapılmıştı. Patolojisi kazeöz nekroz ve granülomatöz infiltrasyon gelmesi üzerine over tüberkülozu tanısıyla olgu 6 ay medikal tedavi almıştı. Olgunun yapılan fizik muayenesinde suprapubik hassasiyet ve normal vulva, anüs ve "hymen" izlendi. Bekâr olması nedeni ile olgunun vajinal değerlendirmesi yapılamadı. USG'de sağ over normal, sol adneksiyal alanda $13 \mathrm{~cm}$ 'lik hematokolposla uyumlu kitle ve çift endometriyal ekojenite izlendi. MRG'de çift uterus, solda $13 \mathrm{~cm}$ 'lik hematokolpos, sol endometrial kavitede sıvı izlendi. Sol endometriyal kavite içerisindeki sıvı pelvisteki hematokolpos ile bağlantılı olarak saptandı (Resim 1, 2). Sol böbrek agenezisi mevcuttu. Sol over izlenmedi. Olgu litotomi pozisyonunda genel anestezi altında operasyona alındı ve solda obstrükte hemivajenin introitustan protrude görünümü izlendi (Resim 3). Obstrükte hemivajen cerrahi insizyonla açlarak hematokolpos boşaltıldı; yaklaşık 1.000 cc hemorajik mayi geldi. Ardından vajinal septum rezeke edildi. Postoperatif izleminde sorun olmayan olgu, önerilerle taburcu edildi.

\section{TARTIŞMA}

Müllerian kanal anomalileri, fetal hayatın 6-9. haftaları boyunca Müllerian kanal gelişimindeki başarısızlığa bağlı olarak ortaya çıkan dişi genital traktın konjenital anomalileridir. Müllerian kanalın gelişimi organogenez, füzyon ve septal rezorpsiyondan oluşan 3 aşamada gerçekleşmektedir. $\mathrm{Bu}$ aşamalardaki bir duraksama Müllerian füzyon anomalilerine neden olmaktadır. Organogenez aşaması kaynaklı patolojiler Müllerian agenezi veya uni-
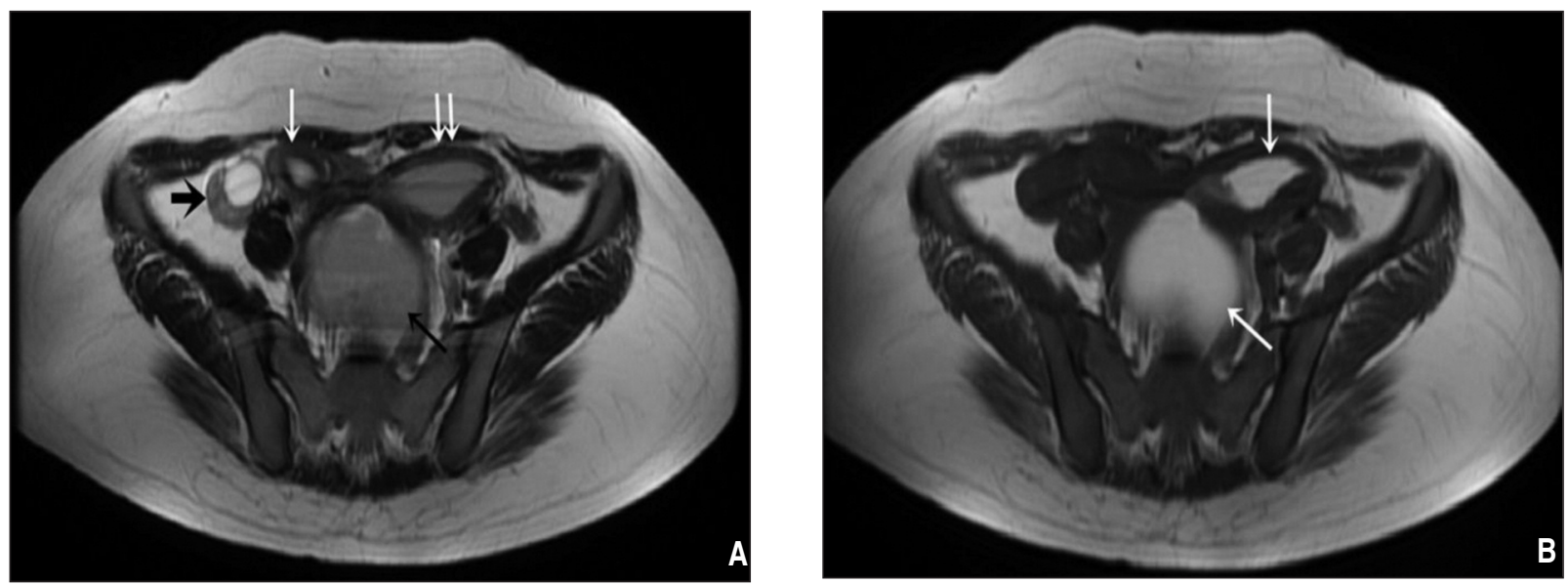

RESiM 1: A. Aksiyel planda T2 ağırlıklı pelvik manyetik rezonans görüntülemede uterus sağ (beyaz ok) ve sol kornuları (çift beyaz ok) birbirlerinden uzaklaşmış, solda daha belirgin olmak üzere her iki endometriyal kavitede sıvı görülmektedir. Siyah ok vajen proksimalindeki sıvııı, çift siyah ok sağ overi göstermektedir. B. Aksiyel planda T1 ağırıklı görüntüde sol kornu ve vajendeki hemorajik sıvı (beyaz oklar) hiperintens izlenmektedir. 

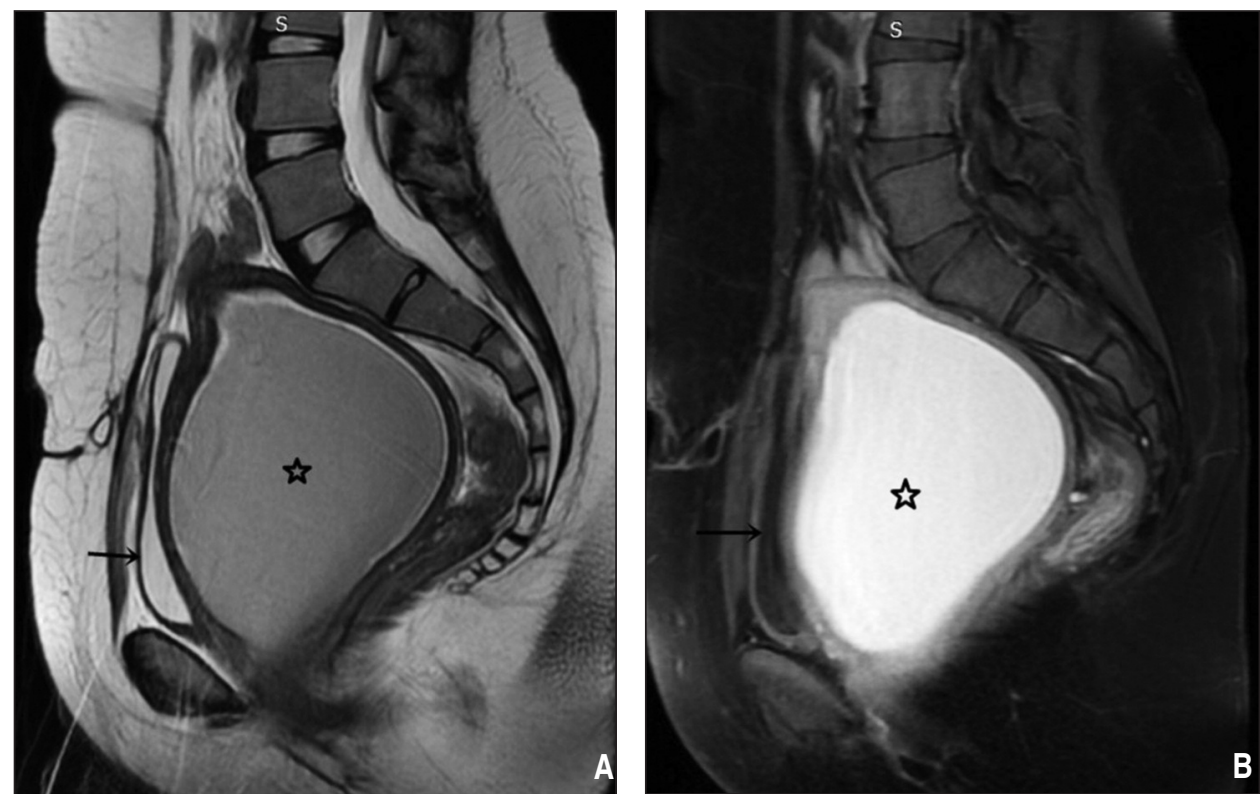

RESiM 2: A. Sagital planda T2 ağırlıklı pelvik manyetik rezonans görüntülemede vajende genişlemeye yol açmış mesane (siyah ok) ve beyin omurilik sıvısına göre hafif hipointens hematokolpos (yıldız) görülmektedir. B. Hematokolpos yağ baskılı T1 ağırlıkı görüntüde hiperintens (yıldız) izlenmektedir.

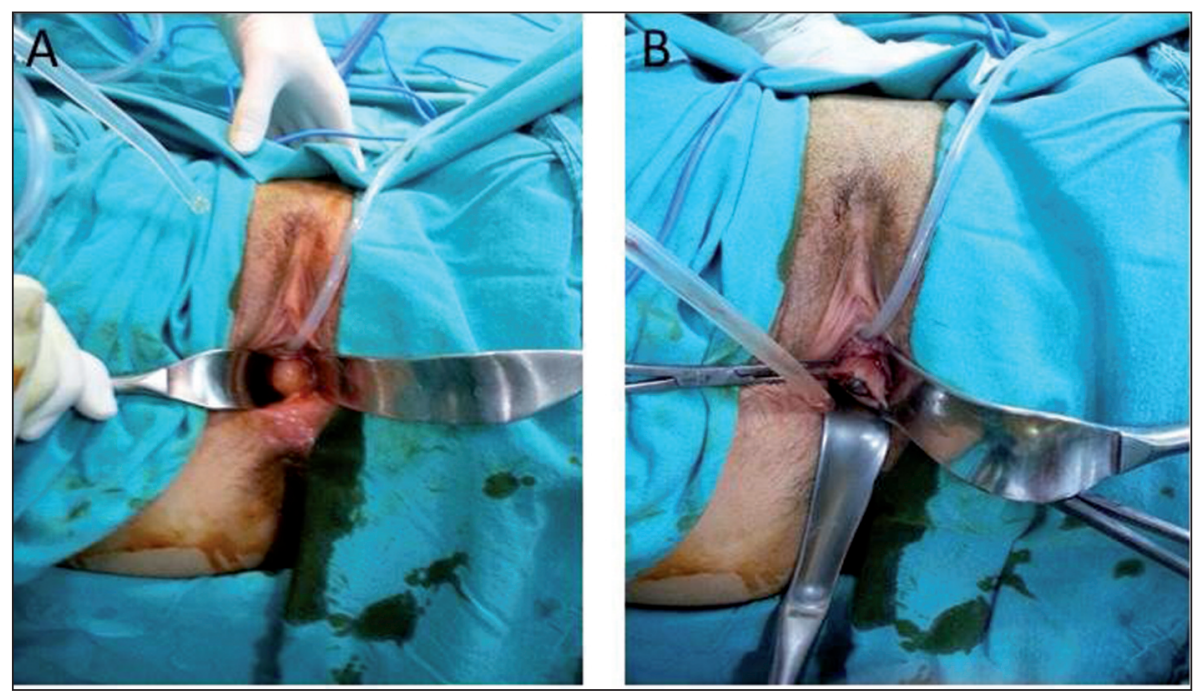

RESiM 3: A. Obstrükte hemivajinanın hematokolposa sekonder olarak vajinal introitustan protrüzyonu. B. Obstrükte hemivajenin drenajı için uygulanan cerrahi insizyon.

kornat uterus; füzyon aşaması kaynaklı patolojiler bikornat uterus veya uterus didelfis; septal rezorpsiyon aşaması kaynaklı patolojiler ise septat uterusa neden olmaktadır. ${ }^{4}$ Müllerian kanal anomalilerine \%20-25 oranında üriner sistem anomalileri de eşlik etmektedir. Wolf kanalı gelişiminde bir kusur mevcut ise o tarafın böbrek ve toplayıcı sistemi gelişemeyeceği gibi, uterusta da füzyon anomalileri meydana gelebilmektedir. ${ }^{5}$ Yaygın olarak kullanı- lan müllerian kanal anomalileri Buttram ve Gibbons tarafından 6 gruba ayrılmıştır. ${ }^{6}$ Obstrüktif hemivajen, uterus didelfis ve ipsilateral renal agenezi birlikteliği HWWS olarak adlandırılmaktadır. ${ }^{1}$ HWWS tip 3 Müllerian anomalinin mezonefrik kanal anomalisi ve vajinal septumla birlikte görülmesidir. Zhu ve ark., HWWS için yeni bir klasifikasyon sistemi tanımlamışlar ve bu klasifikasyon sisteminin, sekonder pelvik endometriyoz ve pel- 
vik inflamasyon gelişmeden sendromun erken tanı ve tedavisinde çok yararlı olacağını savunmuşlardir. ${ }^{7}$

HWWS, genellikle pubertal dönemde menarşı takiben hematokolposa sekonder gelişen şiddetli pelvik ağrı ve pelvik kitle ile kendini göstermektedir. HWWS'li hastalar, bizim olgumuzda da olduğu gibi genellikle normal menstrüasyona sahiptir. HWWS'nin dismenore dışında spesifik bir semptomu yoktur. Çoğu hasta menarşı takiben 2 ay-1 yıl arasında tanı alır. Fizik muayenede sağ tarafta daha sık olmak üzere tek taraflı pelvik kitle saptanır. ${ }^{8}$ Ancak bizim olgumuz, sol obstrükte hemivajen ve sol hematokolposa bağlı pelvik kitle ile prezente olmuştur. Piyohematokolpos, piyosalpenks, piyoperitonit gibi enfektif süreçler, retrograd menstrüasyona sekonder endometriyoz ve pelvik adezyonlar karşılaşılabilecek komplikasyonlar arasındadır. ${ }^{9,10}$ Endometriyoz bu sendromun sık görülen bir komplikasyonudur. Nedeni, obstrüksiyona sekonder meydana gelen retrograd menstrüasyondur. Bu yüzden endometriyozu olan hastalar Müllerian anomali açısından iyi değerlendirilmelidir. $\mathrm{Bu}$ sendromun erken tanı ve tedavisi hastada ileride gelişebilecek sekonder endometriyoz ve infertilitenin önlenmesinde oldukça önemlidir.

Genitoüriner anomaliden şüphelenildiğinde tanıda ilk planda USG kullanılmaktadır. HWWS'de USG değerlendirmesinde proksimal vajina veya uterin serviks seviyesinde sıvı ekojeniteleri ile bağlantılı görünen uterin didelfis, obstrükte hemivajen nedeni ile kavitelerden birinde sıvı koleksiyonu izlenebilir. Bizim olgumuzda olduğu gibi pelviste mikst ekojenitede kistik bir görünüm saptanabilir. ${ }^{3}$ USG'de uterin didelfis ile birlikte anormal vajinal veya uterovajinal sıvı koleksiyonunun saptanması durumunda HWWS akla gelmelidir. Sıvı koleksiyonunun olduğu tarafta böbrek agenezisinin saptanması tanıyı desteklemektedir. Tanıyı kesinleştirmek ve uterovajinal anatomiyi detaylandırmak için pelvis MRG \%100'e yakın doğruluk göstermektedir.,11 MRG, uterus konturlarının, uterin kavitenin ve vajinal septumun şeklinin, sıvı koleksiyonunun natürünün belirlenmesinde, eşlik eden endometrioz, adezyon, inflamasyon gibi komplikasyonların saptanmasında ayrıntılı bilgiler sağlamaktadır. Bu çalış- mada kesin tanısını MRG ile koyduğumuz HWW sendromu olgusu sunulmuştur.

Tedavi seçenekleri içerisinde en çok kullanılan yöntem, bizim de olgumuzda uyguladığımız vajinal septumun rezeksiyonudur. Vajinal septum rezeksiyonu vajinal yolla yapılabileceği gibi hastanın ve ailesinin "hymen"in korunmasını istemesi durumunda histeroskopik olarak da yapılabilmektedir. ${ }^{12} \mathrm{Bu}$ tedavi semptomların düzelmesi ve komplikasyonların önlenmesinde çok etkilidir. Nadir olarak, vajinal septum rezeksiyonunun hematometra gelişimini engellemede yeterli olmadığ durumlarda hemihisterektomi gerekebilmektedir. ${ }^{13}$ Yine, servikal atrezi ile komplike olan hastalarda, septum rezeksiyonu obstrüksiyona bağlı bulguları iyileştirmeyeceğinden hemihisterektomi uygulanmalıdır. ${ }^{7}$ Cerrahi tedavinin uygulanamadığı hastalarda hematometra ve hemato-kolpos gelişimini önlemek amacıyla menstrüasyon süpresif ilaç tedavisi uygulanmalıdır. ${ }^{10}$ Literatürde cerrahi tedavi görmüş hastalarda yaklaşık \%87 oranında fertilite bildirilmiştir. ${ }^{14}$ Gebelik bildirilen opere HWWS hastalarında patolojik gebelik veya gebeliğe bağlı komplikasyon saptanmamıştır. Primer infertilite saptanan hastalarda ise mevcut uterin anomaliden ziyade cerrahi tedavi öncesi retrograd menstrüasyona sekonder gelişen endometriyoz ve intraperitoneal enfeksiyon gibi diğer sebepler düşünülmelidir.

Sonuç olarak; HWWS nadir olarak görülse de şiddetli dismenore ile başvuran ve tedaviye yanıt vermeyen genç yaştaki hastalarda akla gelmelidir. Tanısı semptomlar, fizik muayene ve USG ile konulmakta, MRG ile kesinleştirilmektedir. Vajinal septumun rezeksiyonu, komplikasyonların önlenmesi ve fertilitenin korunmasında en uygun tedavi yöntemidir.

\section{Çıkar Çatışması}

Yazarlar herhangi bir çıkar çatışması veya finansal destek bildirmemiştir.

\section{Yazar Katkıları}

İçerik: Mine İslimye Taşkın, Eda Üreyen; Dizayn: Mine İslimye Taşkın, Erdoğan Bülbül, Ertan Adalı; Metot: Mine İslimye Taşkın, Erdoğan Bülbül; Literatür Araştırması: Mine İslimye Taşkın, Eda Üreyen; Yazan: Mine İslimye Taşkın; Review: Mine İslimye Taşkın, Ertan Adalı. 


\section{KAYNAKLAR}

1. Aveiro AC, Miranda V, Cabral AJ, Nunes S, Paulo F, Freitas C. Herlyn-WernerWunderlich syndrome: a rare cause of pelvic pain in adolescent girls. BMJ Case Rep 2011;2011.pii:bcr0420114147.

2. Zurawin RK, Dietrich JE, Heard MJ, Edwards CL. Didelphic uterus and obstructed hemivagina with renal agenesis: case report and review of the literature. J Pediatr Adolesc Gynecol 2004;17(2):137-41.

3. Yavuz A, Bora A, Kurdoğlu M, Goya C, Kurdoğlu Z, Beyazal M, et al. Herlyn-wernerwunderlich syndrome: merits of sonographic and magnetic resonance imaging for accurate diagnosis and patient management in 13 cases. J Pediatr Adolesc Gynecol 2015;28(1): 47-52.

4. Junqueira BL, Allen LM, Spitzer RF, Lucco KL, Babyn PS, Doria AS. Müllerian duct anomalies and mimics in children and adolescents: correlative intraoperative assessment with clinical imaging. Radiographics 2009;29(4): 1085-103.
5. Acién $P$, Acién $M$. Unilateral renal agenesis and female genital tract pathologies. Acta Obstet Gynecol Scand 2010;89(11):1424-31.

6. Buttram VC Jr, Gibbons WE. Müllerian anomalies: a proposed classification (An analysis of 144 cases). Fertil Steril 1979;32(1):40-6.

7. Zhu L, Chen N, Tong JL, Wang W, Zhang L, Lang JH. New classification of Herlyn-WernerWunderlich syndrome. Chin Med J (Engl) 2015;128(2):222-5.

8. Wu TH, Wu TT, Ng YY, Ng SC, Su PH, Chen JY, et al. Herlyn-Werner-Wunderlich syndrome consisting of uterine didelphys, obstructed hemivagina and ipsilateral renal agenesis in a newborn. Pediatr Neonatol 2012;53(1):68-71.

9. Fedele L, Motta F, Frontino G, Restelli E, Bianchi S. Double uterus with obstructed hemivagina and ipsilateral renal agenesis: pelvic anatomic variants in 87 cases. Hum Reprod 2013;28(6):1580-3.

10. Tong J, Zhu L, Lang J. Clinical characteristics of 70 patients with Herlyn-WernerWunderlich syndrome. Int J Gynaecol Obstet 2013;121(2):173-5.

11. Del Vescovo R, Battisti S, Di Paola V, Piccolo $\mathrm{CL}$, Cazzato RL, Sansoni I, et al. HerlynWerner-Wunderlich syndrome: MRI findings, radiological guide (two cases and literature review), and differential diagnosis. BMC Med Imaging 2012;12:4.

12. Wang J, Zhu L, Lang J, Liu Z, Sun D, Leng J, et al. Clinical characteristics and treatment of Herlyn-Werner-Wunderlich syndrome. Arch Gynecol Obstet 2014;290(5):947-50.

13. Donnez O, Jadoul P, Squifflet J, Donnez J Didelphic uterus and obstructed hemivagina: recurrent hematometra in spite of appropriate classic surgical treatment. Gynecol Obstet Invest 2007;63(2):98-101.

14. Sanghvi $Y$, Shastri P, Mane SB, Dhende NP. Prepubertal presentation of Herlyn-WernerWunderlich syndrome: a case report. J Pediatr Surg 2011:46(6):1277-80. 